\title{
LETTER
}

doi:10.1017/S1041610216001587

\section{The role of standardized instruments in identifying older adults with alcohol problems}

Taylor et al. (2014) raise an important issue concerning the detection of alcohol problems in older adults. The authors identify a number of age-related factors playing a role in the detection of alcohol problems, such as stigma, the concept of alcohol use disorder diagnoses and their standardized assessment, and drinking levels. They list a comprehensive review of tools for assessing alcohol problems among older adults, including laboratory tests, questionnaires, and interviews.

To this list, we would like to add results from a large representative study in six European countries on alcohol problems and alcohol use disorders in primary care (Rehm et al., 2015a). In this study, we used the Composite International Diagnostic Interview (CIDI) to assess alcohol problems considered to be the gold standard for detecting alcohol use disorders (for instance, in the NICE guidelines).

In short, we found that the older the client, the more problems there had been for the CIDI to detect alcohol dependence, taking the diagnosis of general practitioners (GPs) as gold standard (Rehm et al., 2015b). Figure 1 of the cited publication illustrates how both assessments of alcohol dependence were related to age. Of the oldest age group studied, i.e. the patients in primary care between 55 and 64 years of age, the CIDI could only detect $38.6 \%$ of people with alcohol use disorders as diagnosed by the GP (95\% CI: 32.3-44.9\%).

This finding corroborates the conclusion of Taylor et al. (2014) that standardized instruments have problems in identifying people with alcohol use disorders in older adults. In addition to some diagnostic criteria not being applicable to older adults, older adults may no longer recognize or remember their own adaptation processes leading to tolerance - a core diagnostic criterion of alcohol dependence. For a 20 old, the transition from no alcohol to regular alcohol consumption with the inevitable increase of tolerance is easy to remember - as opposed to older adults. In addition, older adults may not recognize loss of control as such and judge their overall social situation as acceptable because of long-term habituation. However, GPs' identification of alcohol use disorders was found to be strongly related to the criterion on continuation of drinking despite social consequences (Web Appendix 4 from Rehm et al., 2015a), which may explain the gap between CIDI and GPs in detecting alcohol use disorders among older adults.

Changing standardized questionnaires/ interviews by including somatic symptoms may improve performance in detecting alcohol problems in older adults (Web Appendix 4 from Rehm et al., 2015a). We also agree with the authors to include questions on drinking levels in order to enhance sensitivity and specificity (Saha et al., 2007). However, from a biological point of view, there are two pharmacokinetic processes that make older people more susceptible to the impact of alcohol: First, as the liver shows reduced degradative activity, more alcohol may reach the blood stream for any given intake; and second, due to on average reduced body water the same amount of alcohol is associated with higher blood alcohol concentration (Ferreira and Weems, 2008). Thus, different norms for detecting problems should be developed.

As such instruments have been, are, and will nevertheless be used in epidemiological research, the resulting incidence and prevalence figures are considerable underestimates. Improved standardized assessments and a combination with clinical judgments from treating GPs may constitute a strategy to obtain more reliable estimates.

\section{Conflict of interest}

JR: reports grants from GWT-TUD during the conduct of the study and grants, personal fees and serving as board member (Nalmefene) for Lundbeck, outside of the submitted work. JM: reports receiving personal fees from Lundbeck, outside of the submitted work.

\section{Description of authors' roles}

Jürgen Rehm is initiator and principal investigator of the APC study and conceptualized this letter. Jakob Manthey is study coordinator, contributed statistical analysis for and reviewed this letter.

\section{Acknowledgments}

The study was financially supported by an investigator-initiated grant given to the last author and the GWT-TUD (Gesellschaft für Wissens- und 
Technologietransfer der TU Dresden mbH company with limited liabilities for transferring knowledge and technology of the Dresden University of Technology) by Lundbeck (grant number 414209). The study sponsor has no role in study design, collection, analysis, or interpretation of data. The study sponsor also had no role in the writing of the report or the decision to submit the paper for publication. The corresponding author confirms that the authors had full access to the data in the study at all times, and had final responsibility for the decision to submit for publication.

\section{References}

Ferreira, M. P. and Weems, M. K. (2008). Alcohol consumption by aging adults in the United States: health benefits and detriments. Fournal of the American Dietetic Association, 108, 1668-1676.

Rehm, J. et al. (2015a). General practitioners recognizing alcohol dependence: a large cross-sectional study in 6 European countries. Annals of Family Medicine, 13, 28-32.

Rehm, J. et al. (2015b). Alcohol dependence and treatment utilization in Euro - a representative cross-sectional study in primary care. BMC Family Practice, 16, 90.
Saha, T. D., Stinson, F. S. and Grant, B. F. (2007). The role of alcohol consumption in future classifications of alcohol use disorders. Drug and Alcohol Dependence, 89, 82-92.

Taylor, C., Jones, K. A. and Dening, T. (2014). Detecting alcohol problems in older adults: can we do better? International Psychogeriatrics, 26, 1755-1766.

JÜRgEN RehM ${ }^{1,2,3,4,5,6}$ AND Jakob MANThey ${ }^{1}$ ${ }^{1}$ Institute for Clinical Psychology and Psychotherapy, TU Dresden, Chemnitzer Str. 46, Dresden 01187, Germany

${ }^{2}$ Centre for Addiction and Mental Health (CAMH), 33

Russell Street, Toronto, ON M5S 2S1, Canada

${ }^{3}$ Campbell Family Mental Health Research Institute, CAMH, 250 College Street, Toronto, ON M5T 1R8, Canada

${ }^{4}$ Institute of Medical Science (IMS), University of Toronto, Medical Sciences Building, 1 King's College Circle, Room 2374, Toronto, ON M5S 1A8, Canada ${ }^{5}$ Department of Psychiatry, University of Toronto, 250 College Street, 8th Floor, Toronto, ON M5T 1R8, Canada

${ }^{6}$ Dalla Lana School of Public Health, University of Toronto, 155 College Street, 6th Floor, Toronto, ON M5T 3M7, Canada 